\title{
2083 Women have higher left ventricular ejection fractions than men: a multivariable analysis in 4864 subjects using SSFP cine MRI Sujata M Shanbhag*1, Erik B Schelbert ${ }^{1}$, Li-Yueh Hsu ${ }^{1}$, Jie J Cao ${ }^{2}$, Sigurdur Sigurdsson ${ }^{3}$ and Andrew E Arai ${ }^{1}$
}

\author{
Address: ${ }^{1}$ National Institutes of Health, Bethesda, MD, USA, ${ }^{2}$ Stony Brook State University of New York, Roslyn, NY, USA and ${ }^{3}$ Icelandic Heart \\ Association, Reykjavik, Iceland \\ * Corresponding author
}

from I I th Annual SCMR Scientific Sessions

Los Angeles, CA, USA. I-3 February 2008

Published: 22 October 2008

Journal of Cardiovascular Magnetic Resonance 2008, I0(Suppl I):A352 doi:10.1 186/1532-429X-10-SI-A352

This abstract is available from: http://jcmr-online.com/content/I0/SI/A352

(c) 2008 Shanbhag et al; licensee BioMed Central Ltd.

\section{Introduction}

Left ventricular ejection fraction (LVEF) is traditionally used as a clinical marker for prognosis and clinical risk stratification. Decisions for prescribing clinical therapies are frequently based on LVEF. The results from existing literature are conflicted with respect to the existence of a gender difference in LVEF. One explanation is that greatly varying magnetic resonance imaging techniques including SSFP, GRE, or hybrid echo planar cine methods were used or that small sample sizes were studied in the publications to date. Thus, it remains unclear whether statistically significant gender-specific differences between LVEF exist.

\section{Purpose}

We retrospectively analyzed the clinical cardiac MRI scans that were performed at the National Institutes of Health, Suburban Hospital, and Icelandic Heart Association since 2001 for gender differences in left ventricular ejection fraction using steady state free precession cine cardiac magnetic resonance imaging.

\section{Methods}

Categorical variables were compared with chi squared test, and continuous variables were compared using Wilcoxon rank sum test. The relation between gender and LVEF was adjusted for covariates using multivariable linear regression models. All cardac MRI scans were performed using SSFP cine cardiac MRI. Variables assessed included gender, age, LVEF, percent LV delayed enhance- ment, LVEDV indexed to BSA, and LVED Mass indexed to BSA.

\section{Results}

We screened over 5000 subjects who had SSFP cine cardiac MRI scans performed and excluded those with missing fields for gender, LVEF, and body surface area (BSA) resulting in 4864 subjects within the collective database. Multivariable analysis showed that delayed enhancement, LVEDV indexed to BSA, age and gender were the strongest predictors of LVEF. However, LVED Mass indexed to BSA was not significantly predictive of LVEF. Since myocardial infarction and EDV were the two strongest predictors of abnormal ejection fraction, we excluded all patients with delayed LV enhancement and LVEDV/BSA exceeding two standard deviations beyond the normal accepted values for SSFP cine techniques in cardiac magnetic resonance imaging of normal subjects. In the remaining 3223 patients (1829 men and 1394 women), women subjects still had a significantly higher ejection fraction compared to men $(63 \%$ versus $60 \%, \mathrm{p}=<0.001)$.

\section{Conclusion}

To the best of our knowledge, this is the largest study to date that used SSFP cine MRI and manual planimetry by cardiologists to measure gender related differences in LVEF. Despite the referral nature of the population, a group of 3223 subjects had diagnostic imaging to exclude the most common causes of abnormal LVEF. Thus, in 
both overall multivariable analysis and in the subset of patients without CAD or dilated cardiomyopathy, women were found to have a higher LVEF than men.

Publish with Biomed Central and every scientist can read your work free of charge

"BioMed Central will be the most significant development for disseminating the results of biomedical research in our lifetime. " Sir Paul Nurse, Cancer Research UK

Your research papers will be:

- available free of charge to the entire biomedical community

- peer reviewed and published immediately upon acceptance

- cited in PubMed and archived on PubMed Central

- yours - you keep the copyright 